\title{
Comparing Human-Robot Proxemics between Virtual Reality and the Real World
}

\section{RUI LI}





\section{Comparing Human-Robot Proxemics between Virtual Reality and the Real World}

Master of Science in Media Technology, Master's program in Computer Science

June 25, 2018

\section{Author}

Rui Li

rui3@kth.se

\section{Supervisors}

Iolanda Leite

Examiner

Christopher Peters 



\section{Abstract}

Virtual Reality (VR) is gaining more and more popularity as a research tool in the field of Human-Robot Interaction (HRI). To fully deploy the potential of VR and benefit HRI studies, we need to establish the basic understanding of the relationship between the physical, real-world interaction (Live) and VR. This study compared Live and VR HRI with a focus on proxemics, as proxemics preference can reflect comprehensive human intuition, making it suitable to be used to compare Live and VR. To evaluate the influence of different modalities in VR, virtual scenes with different visual familiarity and spatial sound were compared as well. Lab experiments were conducted with a physical Pepper robot and its virtual copy. In both Live and VR, proxemics preferences, the perception of the robot (competence and discomfort) and the feeling of presence were measured and compared. Results suggest that proxemic preferences do not remain consistent in Live and in VR, which could be influenced by the perception of the robot. Therefore, when conducting HRI experiments in VR, the perceptions of the robot need be compared before the experiments. Results also indicate freedom within VR HRI as different VR settings are consistent with each other. 


\title{
Comparing Human-Robot Proxemics between Virtual Reality and the Real World
}

\author{
Rui Li \\ KTH Royal Institute of \\ Technology \\ Stockholm, Sweden \\ Rui3@kth.se
}

\begin{abstract}
Virtual Reality (VR) is gaining more and more popularity as a research tool in the field of HumanRobot Interaction (HRI). To fully deploy the potential of VR and benefit HRI studies, we need to establish the basic understanding of the relationship between the physical, real-world interaction (Live) and VR. This study compared Live and VR HRI with a focus on proxemics, as proxemics preference can reflect comprehensive human intuition, making it suitable to be used to compare Live and VR. To evaluate the influence of different modalities in VR, virtual scenes with different visual familiarity and spatial sound were compared as well. Lab experiments were conducted with a physical Pepper robot and its virtual copy. In both Live and VR, proxemics preferences, the perception of the robot (competence and discomfort) and the feeling of presence were measured and compared. Results suggest that proxemic preferences do not remain consistent in Live and in VR, which could be influenced by the perception of the robot. Therefore, when conducting HRI experiments in VR, the perceptions of the robot need be compared before the experiments. Results also indicate freedom within VR HRI as different VR settings are consistent with each other.
\end{abstract}

\section{INTRODUCTION}

Virtual Reality (VR) is gaining more and more popularity as a research tool in the field of HumanRobot Interaction (HRI) [1][2][3][4]. VR has been used to test teleoperation and collect demonstration data to train machine learning algorithms, which showcased the effectiveness of learning visuomotor skills using data collected by consumer-grade devices [1]. VR teleoperation systems were proposed to crowdsource robotic demonstrations at scale [2]. A VR simulation framework was also proposed to replace the physical robot, as VR can enable high level abstraction in embodiment and multimodal interaction [3]. VR has also been used as a rapid prototyping tool to design in-vehicle interactions and interfaces for self-driving cars, which showed the evocation to genuine responses from test participants [4].

Compared to other HRI experiment methods, VR as an emerging interactive media provides unique advantages. VR HRI has the potential of having higher immersion and fidelity than picture based HRI, video-based HRI and simulated HRI. In situations where the perception of the robot is challenging, compared to on-screen viewing, VR display showed significant improvement on collaborative tasks [5].

When comparing VR HRI to the physical, realworld interaction (Live HRI), there is a trade-off between the two. VR experiences still cannot replace physical experiences due to system limitation, and limited interaction modalities etc. [6]. For example, system limitations such as limited field of view and low display resolution could reduce immersion and presence of the VR experience, resulting in different behaviors from Live experiments. Limited interaction modalities, such as the absence of touch, means that the participant could not feel the robot or even go through the robot, which could potentially break the entire interaction.

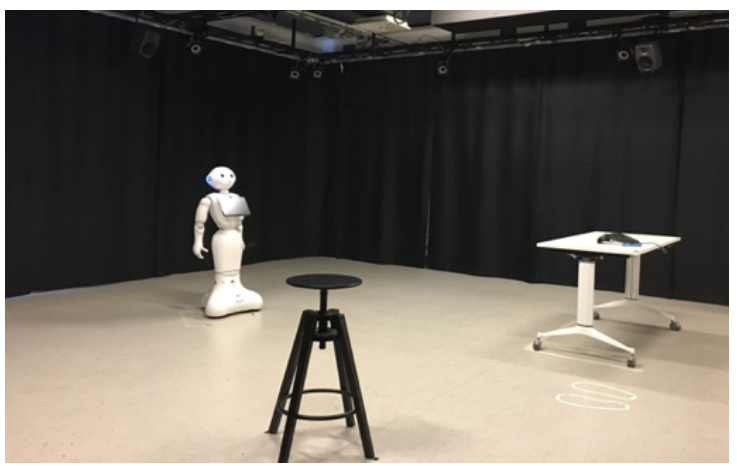

Figure 1: Photograph of the Live experiment setting

However, with the help of the distribution of consumer-grade VR devices and online crowdsourcing platforms, VR HRI has the potential to gain massive data for training robotic behavior and studying HRI related issues. Data collection through VR can also reduce noise and improve the data quality [1], which help to ease data processing and algorithm training. Furthermore, VR HRI experiments can test concepts and interactions without physical robots, making it more resource efficient and less expensive than Live HRI. Less hardware also means that the experiment will be less cumbersome to set up, easier to be reproduced and to ensure experiment quality. 
In this study, HRI Proxemics (the preferred personal space between a human and a robot) was compared to give a better justification and more basic understanding of the relationship between Live and VR. Proxemics preferences rely on lower level intuition [7], therefore, reflect the differences in the perceptions between Live and VR better. Compared to other HRI subject such as conversational (audio) or gaze behavior (visual), which are more modality dependent, proxemics can give a comprehensive understanding of the human responses.

In addition, variations of modalities in VR can greatly influence human perception. For example, a higher visual familiarity of the physical environment in VR can decrease the effect of distance distortion [8]. Auditory inputs play another important role in VR, the addition of spatial sound can increase the sense of presence in VR and provide sound localization [9]. Thus, this work also compares VR settings with variance in modalities to evaluate the impacts of visual familiarity and spatial sound on VR HRI experiments.

A $2 \times 3$ mixed design experiment was conducted to evaluate the differences between Live and VR HRI, as well as the influence of visual familiarity and spatial sound in VR. For the Live HRI, the pepper robot from Softbank Robotics was used (Figure 1). In the VR HRI, a 3D model of the same robot was used. To measure visual familiarity, the VR scene was created in Blender based on a 3D scan of the physical lab. The spatial sound was created by enabling the movement of the physical robot, due to the difficulties of engineering spatial sound. The interaction was implemented in Unity.

As an objective measurement for proxemics preference, the minimum comfort distance (MCD) was measured. In addition, for the psychological perception of the experience, the feeling of presence was measured with the SUS questionnaire. For the perception of the robot, two relevant factors, competence and discomfort was measured with the ROSAS questionnaire.

\section{RELATED WORK}

\section{VR in HR}

Before the popularity of consumer-grade head mounted display (HMD), research has been done to compare Live and VR HRI in a Cave Automatic Virtual Environment (CAVE) [10]. CAVE consists of a cubed display with four screens in the forward, right, left, and downward directions. Participants wore polarized glasses with magnetic sensors, and, while they were in the cube, they saw $3 \mathrm{D}$ images based on the position in which they stood. The study showed no significant difference regarding desired personal space between Live and VR, but the VR robot was perceived as being more under human control than the real robot. It was also perceived to have lower utility and possibility of communication than the real robot. However, the CAVE VR system is quite different from current consumer-grade HMD VR devices, calling further research to examine the differences between Live and VR HRI with HMD VR systems.

With consumer-grade HMD, VR has been tested as a teleoperation interface[1], HRI experiment tool [4] and data collection tool [2]. Research has also been done to compare how human gave natural language instructions towards a VR teleoperated humanoid robot and towards another human [11]. It was suggested that humans will use politeness strategies equally with human and teleoperated robotic teammates. However, human-teleoperated robots were perceived as less intelligent than human teammates.

In conclusion, current VR related HRI research illustrated great potential of VR as a research method for HRI. However, to deploy VR as a tool for conducting HRI experiments and collecting massive training data, more up-to-date theoretical ground about the relationship between consumergrade VR and Live HRI studies still needs further research.

\section{Proxemics in HRI}

Proxemics, the personal space that people maintain around themselves, introduced by Hall in 1966 [7], was extensively studied in the last decades. The four personal space zones as shown in Table 1 (intimate, personal, social and public) are assumed to hold in general for people.

\begin{tabular}{l|l|l} 
Zone & Range $(\mathrm{m})$ & Situation \\
\hline Intimate & $0-0.45$ & Close friend or lover \\
Personal & $0.45-1.2$ & Conversation between friends \\
Social & $1.2-3.6$ & Conversation \\
Public & $>3.6$ & Public speaking
\end{tabular}

Table 1: The four personal space zones as defined by Hall (1966).

Proxemics is comparably well studied in HRI. Similar to the personal space between humans, people tend to maintain their personal zone when interacting with robots [12] [13] [14] or even virtual robots [15].

For example, in controlled experiments with children and adults interacting with the mechanistic robot PeopleBot, children tended to stand further away from the robot than adults. Research concerning the dynamic interaction of people teaching robots to identify objects suggested that adults generally prefer to maintain a personal distance $(0.45-1.2 \mathrm{~m})$ from the robot by the definition of Hall [7], although actual distance varied by the type of task (i.e., following, showing, and validating missions) [16]. Previous research also suggested that personal experience with pets and robots decreases a person's personal space around robots [13]. In a stop task carried out with a 
humanoid robot ASIMO in both Live and VR, Kamide et al. [10] showed that the preferred personal space was around $0.8( \pm 0.1) \mathrm{m}$.

These studies highlighted the importance for the robot to respect the personal space of the human. Moreover, proxemic preferences can reflect comprehensive human intuition between Live and VR, thus this research chose to focus on the context of HRI proxemics.

\section{Comparison between Reality and Virtual Reality}

To measure the differences between Live and VR HRI tasks, it is first important to know how to quantify the differences between physical and virtual experiences. Numerous research has been done to investigate the difference between virtual and physical experiences since the early 1990s [17].

Research [6] distinguish the two concepts of immersion (an objective description of aspects of the system such as field of view and display resolution) and presence (a subjective phenomenon such as the sensation of being in a virtual environment). For an HRI experiment with consumer devices, it is important to assess the level of presence (whether people felt being in the experiment environment and act and think the same way) in VR as in Live.

There is not yet a commonly accepted paradigm for the assessment of presence. In general, presence can be measured subjectively through questionnaires or objectively through physiological and behavior measures etc. [17].

Concerning the context of HRI experiments, it is the subjective evaluation of presence that matters, therefore, questionnaires were used to measure presence in this research. However, most of the presence questionnaires are only designed to measure presence in VR, making them not suitable to compare Live and VR. M. Usoh, E. Catena, S. Arman, and M. Slater [18] proposed that presence questionnaires should pass a "reality check" and result in higher presence in Live than in VR. They compared the Presence Questionnaire and the Slater-Usoh-Steed (SUS) Questionnaire. Results suggested that SUS is superior than the Presence Questionnaire in showing the difference between VR and reality. Therefore, the SUS questionnaire was used to evaluate presence for this research.

\section{Visual familiarity in VR}

Numerous previous studies have suggested that distances appear to be compressed in VR, relative to in the real world [19][20].

Since proxemic preferences is closely related to one's distance perception, it is worth well to reexamine if HRI proxemic preferences will be difference via HMD.

Surprisingly, [8] showed that by making the virtual environment (VE) closely resemble of the physical environment, the perceived distance might not be distorted. The visual familiarity with the physical world is manipulated in the VR scene.

\section{Spatial sound and VR}

Spatial Sound has been accepted as a significant cue for localizing the sound origin and increasing presence in VR.

The sense of presence was investigated as a function of the addition or absence of spatial auditory cues during a navigation task within a stereoscopic VE, results indicated that the addition of spatialized sound significantly increased the sense of presence [21]. Similarly, [22] investigated the effects of tactile, olfactory, audio and visual sensory cues on a participant's sense of presence in a VE and on their memory for the environment. Results indicated that the addition of tactile, olfactory and auditory cues to a VE increased the user's sense of presence and memory of the environment. Surprisingly, increasing the level of visual detail did not result in an increase in the user's sense of presence or memory of the environment.

[9] examined whether three-dimensional reproduced sounds increase the sense of presence in auditory VEs by using physiological and psychological measures and found that presence ratings for spatialized sounds were greater than for nonspatialized sounds.

In the context of HRI proxemics, spatial sound plays another important role as it enables sound localization, the listener's ability to identify the location or origin of a detected sound in direction and distance [23]. It is reasonable to hypothesize that the absence or addition of spatial sound can influence proxemic preferences in VR. Therefore, one of the focuses of this study is to investigate the influence of spatial sound on proxemic preferences in VR.

\section{RESEARCH HYPOTHESES}

Based on the existing literature, this study developed three main manipulations: the presentation methods (Live vs. VR), the visual familiarity of the physical environment in VR and spatial sound. To test the influence of these manipulations, the following research hypotheses were made for this study.

\section{Comparison between Presentation methods}

- H1: Since distances appear to be compressed in VR via HMD systems [19][20], it is hypothesized that proxemic preferences will be different between Live and VR.

- H2: Since previous research suggest that presentation methods can influence the perception of the robot [5] [8], it is hypothesized that different presentation methods will influence the perception of the robot between Live and VR. 
- H3: Since in general, the feeling of presence is higher in reality than in VR [18], it is hypothesized that participants will have a higher feeling of presence in Live than in VR.

Manipulation of visual familiarity and spatial sound in VR

- H4: Since higher visual familiarity with the physical world can help to enhance presence and to construct space perception in VR [9], it is hypothesized that visual familiarity can reduce the difference in proxemic preferences between the two presentation methods.

- H5: Since spatial sound can increase presence and provide sound localization to determine the distance of the sound source [23][9], it is hypothesized that the addition of spatial sound in VR can reduce the difference in proxemic preferences between Live and VR.

\section{METHOD}

\section{Experimental Design}

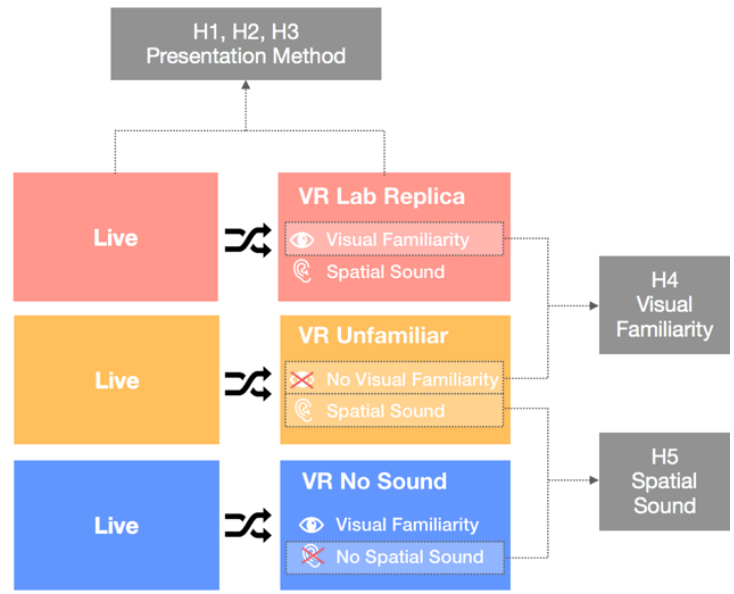

Figure 2: Schematic representation of the experiment design.

A 2 × 3 mixed design experimental setup was used to test the hypotheses (Figure 1). The within variable is the presentation method, i.e. the live environment where a physical robot was present (Live), and the mediated virtual environment ("VR") in which a virtual model of the same robot was present. Each participant took part in two trials, one "Live trial" and one "VR trial", in a randomized order to avoid bias. The within-subject experiment contributed to $\mathrm{H} 1, \mathrm{H} 2$ and $\mathrm{H} 3$, examining the influence of presentation methods on the minimum comfort distance (MCD), the perception of the robot and the feeling of presence. Between subjects, participants were separated into three groups, in which the VR trials were altered to explore the influence of visual familiarity and spatial sound (Figure 1). The comparison between VR Lab Replica condition and

\footnotetext{
${ }^{1}$ www.ald.softbankrobotics.com/en/robots/pepper
}

VR Unfamiliar condition contributed to H4: visual familiarity can reduce MCD difference between the two presentation methods and increase the feeling of presence. The comparison between VR Lab replica condition and VR No sound condition contributed to H5: The addition of spatial sound can help to reduce MCD difference between the two presentation methods and increase the feeling of presence.

\section{Participants}

63 participants with English proficiency were recruited through convenience sampling. They were rewarded with a movie voucher after participation. Four participants were excluded due to failure in completing the questionnaires. Our final sample included 60 participants ( 27 females, 33 male), with age range from 20 to $34(\mathrm{M}=24.22, \mathrm{SD}=$ 2.37). Participants were randomly assigned to one of the three between-subject groups, mixing age and gender. The Lab Replica group contained 12 females and 9 males $(\mathrm{M}=23.67, \mathrm{SD}=1.96)$, the Unfamiliar group consisted of 9 females and 11 males (M $=23.95$, $\mathrm{SD}=1.70$ ), and the No Sound group contained 6 females and 13 males $(\mathrm{M}=25.11, \mathrm{SD}=$ 3.13).

\section{Implementation}

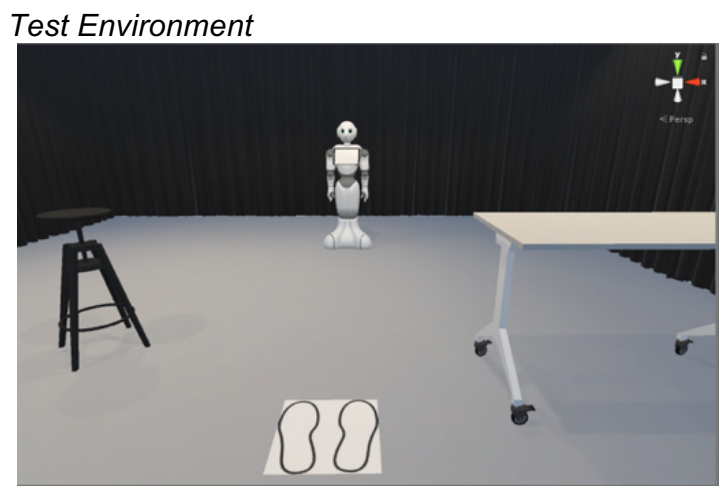

Figure 3: Screenshot the replicated scene of the Live environment in VR

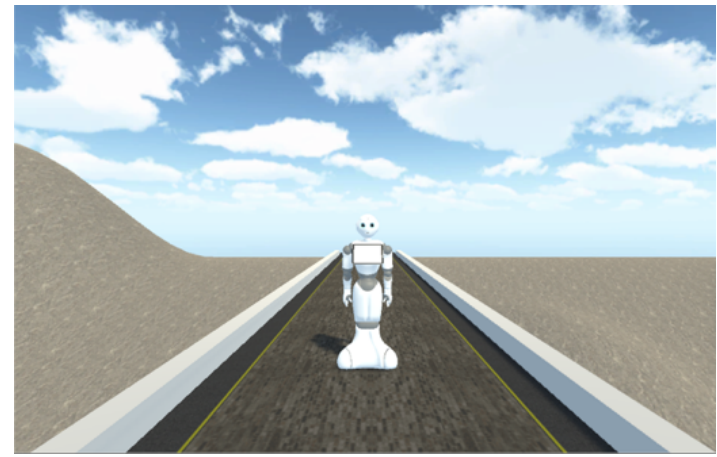

Figure 4: Screenshot of the unfamiliar VR scene

The study was conducted at the PMIL lab on Kungliga Tekniska Högskolan (KTH) campus with the Pepper robot from Softbank Robotics ${ }^{1}$, which is 
a $1.2 \mathrm{~m}$ high, mobile humanoid robot (Figure 2). The VR HRI simulation was developed in $\mathrm{Unity}^{2}$ with a rigged 3D model of the Pepper Robot. HTV Vive ${ }^{3}$, a consumer-grade VR system, was used to emerge participants into VR. The HTC Vive HMD has a nominal field of view of about $110^{\circ}$ (approximately $90^{\circ}$ per eye) through two $1080 \times 1200$ pixel displays that are updated at $90 \mathrm{~Hz}$. The replicated scene of the live environment was created based on the $3 \mathrm{D}$ scan of the PMIL lab in Blender ${ }^{4}$, Figure 2 and Figure 3 compares the real lab environment and the virtual lab replica. The unfamiliar VR scene is an outdoor scene with the Pepper robot standing on the road, as shown in Figure 4.

\section{HRI design}

A stop task was used to measure the MCD between the participant and the robot [24]. Generally, two tasks are performed in the stop task. The participants are instructed to approach the robot and the participants are instructed to stand still and let the robot approach them. However, only the second task was used in the experiment: letting the robot approach the human. As recent studies have shown that whether being approached by the robot or approaching the robot does not have an influence on proxemics preferences [13][10]. Furthermore, the influence of spatial sound cannot be compared in the task of a human approaching a robot since there will be no motor movement of the robot, thus will be no sound. To test the influence of spatial sound in VR, only the human approaching the robot task was chosen.

The interactions in Live and VR were kept consistent for the sake of comparison: the participants hold the HTC Vive controller and can pull the trigger of the controller to enable robot moving forward. When they feel that the robot was too close, they can pull the trigger again to stop the robot.

All communication between the robot and Unity was sent through a wireless local area network (WLAN) created via Choregraphe ${ }^{5}$, a software to visually program the behavior of Pepper. In the network, Choregraphe acted as the server while Pepper and Unity were both the clients. To illustrate, in Live setting, when the trigger is pulled, a signal is sent from the Vive Controller through Unity and Choregraphe to the robot via WLAN. In VR, when the triggered is pulled, the signal will only be sent to Unity, instructing the virtual robot to move forward.

\footnotetext{
${ }^{2}$ www.unity3d.com

${ }^{3}$ www.vive.com/

${ }^{4}$ www.blender.org

${ }^{5} \mathrm{http}: / /$ doc.aldebaran.com/1-

14/software/choregraphe/choregraphe_overview.html
}

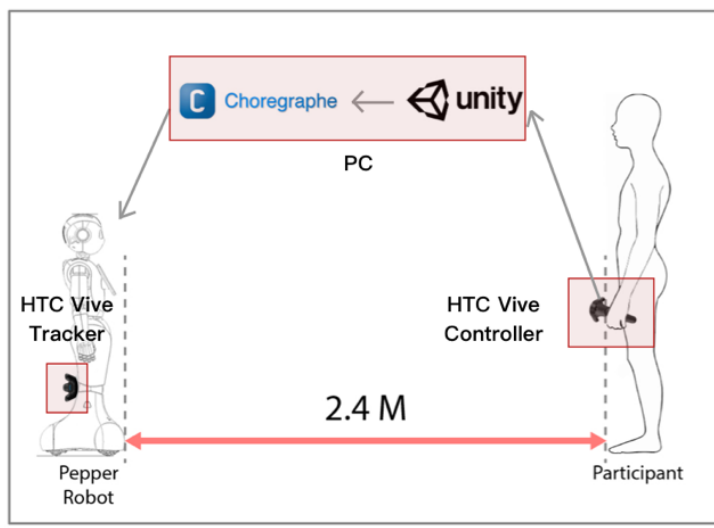

Figure 5: Illustration of experiment setting

In the VR trials with spatial sound, the sound was created by enabling the real robot to move at the same time with the virtual robot, generating motor sounds in the same way as in the Live trials. The real robot was used to create spatial sound due to the complexity of engineering spatial sound [23]. To synchronize the physical robot with the movement of the virtual robot, the 3D model of the robot was attached to the movement of the HTC Vive tracker ${ }^{6}$ placed at the back of the real robot (Figure 5). The tracker can track the location of the physical robot and send it back to VR.

In the VR trials without spatial sound, the movement of the robot was programmed to follow a prerecorded simulation of the movements tracked with the physical robot.

\section{Measurements}

\section{Proxemics}

The location of the robot was documented via the same HTC Vive tracker, that was placed on the back of the physical robot. By attaching the tracker to the robot, the location of the robot can be sent to Unity via the tracker. As a backup, the location data of the robot was recorded as well via the Magnetic Rotary Encoders inside the Pepper robot that could be accessed via the Locomotion control $\mathrm{API}^{7}$. When the participant pulls the trigger to start the robot, the system will $\log$ the current location of the robot as the starting point. When the participant pulls the trigger again to stop the robot, the system will log the current location of the robot as the ending point. For each trial, the displacement of the robot was computed with the starting and ending location point of the robot. Since the robot always started at the same spot $(2.4 \mathrm{~m}$ from the participant), the MCD of

\footnotetext{
${ }^{6}$ https://www.vive.com/eu/vive-tracker/

${ }^{7}$ http://doc.aldebaran.com/2-4/naoqi/motion/controlwalk.html
} 
the trial was computed as " $2.4 \mathrm{~m}$ minus displacement of the robot".

\section{Perception of the robot}

The Robotic Social Attributes Scale (RoSAS) was used to evaluate the perception of the robot. RoSAS is an empirically validated method of measuring the perception of the robot and has three factors: competence, discomfort and warmth [25]. For this study, only the competence and discomfort factors were measured, since the warmth factor is not related to the task. Participants were asked to rate how closely each of the 12 items is associated with the robot (real or virtual) they encountered in the task they just performed. Ratings were on a scale from 1 to 7 where 1 was 'not at all', 4 was 'a moderate amount', and 7 was 'very much so'. The same set of RoSAS questionnaire was used for the entire experiment, with each item presented in a randomized order.

\section{Presence}

The SUS questionnaire, which comprises six questions, was used to measure presence in this study. Participants were asked to rate the questions based on a 7-point Likert scale. The SUS questionnaire used to measure VR trial is shown in Appendix 1. To measure Live trial, the direct reference of "Virtual Environment" in the questionnaire is replaced by "the lab" [18]. Similar with the ROSAS questionnaire, items of the SUS were presented in a randomized order.

\section{Procedure}

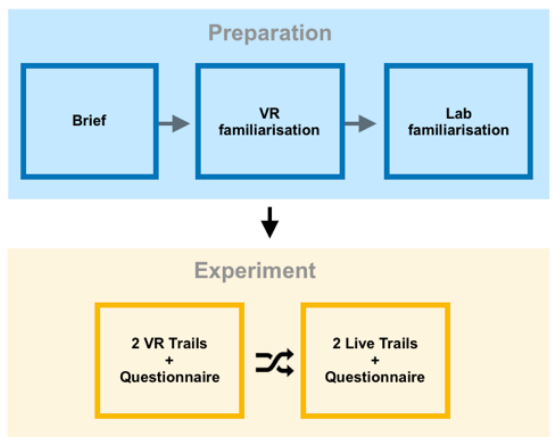

Figure 6: Schematic illustration of the experiment procedure

For preparation, all participants were briefed that the experiment is about human robot interaction in both reality and virtual reality, then asked to sign the informed consent. Then, a VR familiarization session and a lab familiarization session were performed (Figure 6). The VR familiarization session requires that participant to look and move around in a VR scene and count the number of colored rocks. The purpose of the VR familiarization session is to help the participants reduce the novelty effect and getting familiar with VR, since their experience with VR may vary. In the lab familiarization session, participants were asked to walk around the lab and get familiar with the physical space and enhance their spatial perception of the physical lab. To calibrate the spatial perception, participants were also asked to put the controllers on the stool and the desk (see Figure 2) in the physical world, and recollect the controllers once they were in VR.

After the preparation, the participant took part in both the VR and Live trials in randomized order. In the Live trail, the robot was placed at a fixed position (marked on the floor in the lab) $2.4 \mathrm{~m}$ from the participant, which was directly facing the participant as shown in Figure 4. Then, the researcher explained the interaction mechanism and invited the participant to practice the interaction once. After making sure that the participant was comfortable with the interaction mechanism, the researcher read out the aim of the trial and announced that the participant can start. The participant pulled the trigger to start the robot, and pulled the trigger again when they felt that the robot was too close and making them feel uncomfortable. In the VR trail, the participant was asked to follow the exact same procedure but while wearing an HMD that would immerse him/her in a VE with different characteristics depending on the experimental group. The different settings of the three between subject groups are explained in Figure 1. In both VR and Live, the trials were performed twice for the sake of reliability.

After each Presentation Method was tested, the participant was administrated the presence questionnaire (SUS) [18] and the Perceived Competence and Discomfort questionnaire (ROSAS) [25]. At the end of all trials, demographic information was collected through questionnaire. Once the participant completed all sessions, they were debriefed about the purposes of the study and discuss the study with the researcher.

\section{RESULTS}

T-tests were used for the planned pairwise comparisons of the established hypotheses. Internal consistency reliability was assessed for the relevant ROSAS factors using Cronbach's alpha. Results indicate high internal consistency for both discomfort (live trial $\alpha=.81$, VR trial $\alpha=.84$ ) and competence (live trial $\alpha=.82$, VR trial $\alpha=.80$ ). Therefore, the mean of each factor was computed to be used for the T-tests.

\section{The influence of Presentation Methods}

A paired-samples T-test was conducted to evaluate the impact of the Presentation Method (Live vs. VR) on MCD and the perception of the robot.

\section{Proxemics}

The mean distance of the trials was computed from the two trials as the final mean MCD. Figure 7 shows the differences in the mean MCD between Live and VR. The chart shows that the robot came closer to people in Live than in VR. The result of T- 
test confirmed with this observation. There was a significant difference for the mean of preferred distance, $\mathrm{t}(20)=-4.44, \mathrm{p}<.0005, \eta^{2}=.50$. The MCD in Live trials $(\mathrm{M}=.29, \mathrm{SD}=.18)$ was significantly shorter than in VR trials $(\mathrm{M}=.43, \mathrm{SD}=.46)$. However, both means of preferred distance fell into the intimate zone $(0-0.45 \mathrm{~m})$ based on the definition of Hall [7], which is also in line with previous research on HRI Proxemics [13]. In conclusion, our results confirmed $\mathrm{H} 1$, indicating that Presentation Method has an influence on personal space between human and robot.

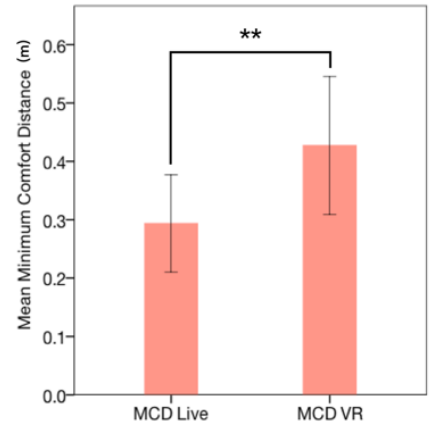

Figure 7: The influence of Presentation Method on the MCD. $(* *)$ denotes $p<.005$. The personal space was smaller in Live than in VR.

Perceived discomfort and perceived competence

Figure 8 shows the differences in the mean scores of competence and discomfort factors from the ROSAS questionnaire. As we can observe, the chart to the left showed that Live or VR did not influence the perceived competence of the robot. The chart to the right shows that the robot is perceived to be more discomforting in Live than in VR. Aligned with the results of proxemic preferences, there was a significant difference in perceived discomfort, $\mathrm{t}(20)$ $=-2.52, \mathrm{p}<.05, \eta^{2}=.24$, with the $\mathrm{VR} \operatorname{robot}(\mathrm{M}=2.13$, $\mathrm{SD}=1.21$ ) being perceived to be more discomforting than the Live robot $(M=1.87, S D=1.04)$. There was no statistically significant difference in perceived competence between Live and VR, t $(20)=-0.50$, $p=.62, \eta^{2}=.01$. These results are in line with previous research, as a significant difference for the psychological evaluation of the robot perception between Live and VR was found [10]. In conclusion, our results confirmed $\mathrm{H} 2$ : suggesting that Presentation Method has an influence on the perception of the robot.
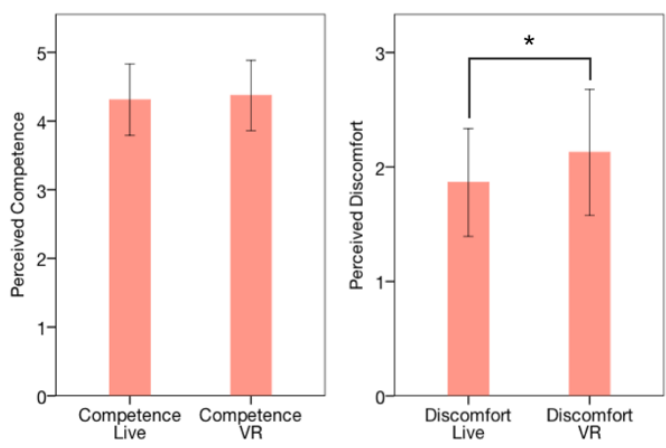

Figure 8: The influence of Presentation Method on the perceived competence and perceived discomfort (right), (*) denotes $p<.05$. The graph on the left showed that Live or VR did not influence the perceived competence, the graph on the right showed that the robot is perceived to be more discomforting in Live than in VR

\section{Presence}

An overall presence score was computed by summing up the count of high (score ' 6 ' or ' 7 ') responses for each one of the 6 questions as recommended for the SUS questionnaire [26]. Figure 9 illustrates the differences in the presence scores between Live and VR from the SUS questionnaire. As shown in the chart, the presence score in Live is much higher than in VR. A logistic regression was performed to ascertain the effect of Presentation Method on presence following the method intended for SUS [26]. Treating the SUS presence score as binomially distributed for a logistic regression on Presentation Method, there was a significant difference between Live and VR, $\mathrm{X}^{2}(1, \mathrm{~N}=40)=12.13, \mathrm{p}<.0005$. This result confirmed $\mathrm{H} 3$, indicating that participants had a higher feeling of presence in Live than in VR. It also suggested that the SUS questionnaire can distinguish Live and VR, aligned with previous research results using the same metric [18]. In conclusion, our results gave support to $\mathrm{H} 3$ : suggesting that people have a higher feeling of presence in Live than in VR.

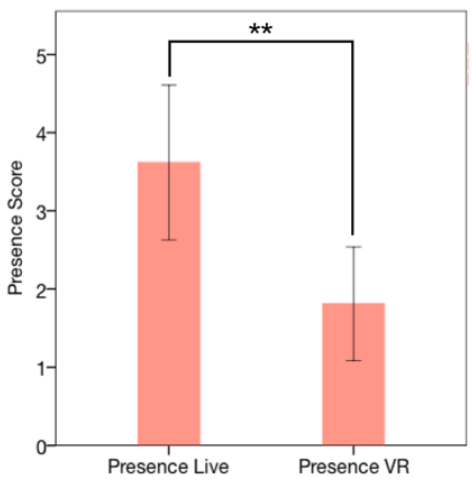

Figure 9: The influence of Presentation Method on presence, $\left({ }^{* *}\right)$ denotes $p<.005$. Participants had a stronger feeling of presence in Live than in VR.

\section{The influence of visual familiarity}

Hypothesis 4 predicted that the addition of visual familiarity can increase presence and reduce the differences in MCD between Live and VR. Figure 10 (Left) shows the MCD in both Live and VR for the Unfamiliar and Lab Replica Condition. Conflicting with the prediction of $\mathrm{H} 4, \mathrm{MCD}$ difference between Live and VR was slighter larger in the Lab Replica than in the Unfamiliar condition. However, an independent T-test between the MCD difference in VR Lab Replica and VR Unfamiliar showed no significance, $\mathrm{t}(39)=1.09, \mathrm{p}=.28, \eta^{2}=$ .03 . 
Figure 10 (Right) shows the presence score for Unfamiliar VR and Lab Replica VR from the SUS questionnaire. Contrary to H4, Lab Replica VR showed slightly lower presence than Unfamiliar VR. However, a logistic regression showed no significance difference in presence between Unfamiliar and Lab Replica, $\mathrm{X}^{2}(1, \mathrm{~N}=41)=.81$, $\mathrm{p}$ $=.37$. Therefore, results did not provide support for $\mathrm{H} 4$, indicting that visual familiarity does not influence presence nor the difference in proxemic preferences between Live and VR.
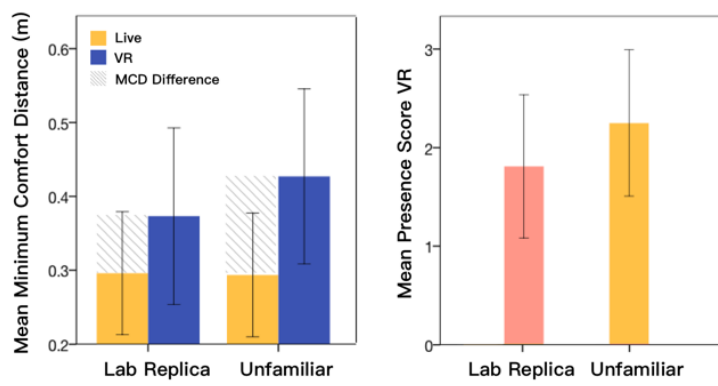

Figure 10: (Left) The influence of visual familiarity on presence. (Right) The influence of visual familiarity on MCD.

The influence of spatial sound

Hypothesis 5 predicted that the addition of spatial sound in VR can reduce MCD difference between Live and VR. Figure 11 (Left) shows the MCD in both Live and VR for the Lab Replica and No Sound condition. Contrasting to $\mathrm{H} 5$, we could observe that the MCD difference in NO Sound condition is slightly smaller than in Lab Replica, although an independent T-test showed no significance between the two, $\mathrm{t}(38)=.96, \mathrm{p}=.35, \eta^{2}=.02$.
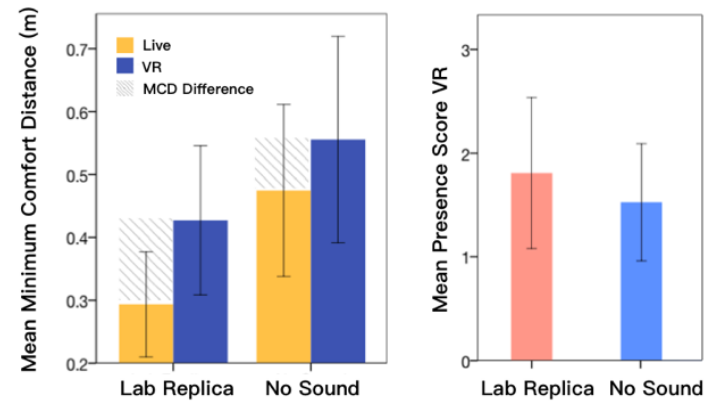

Figure 11: (Left) The influence of spatial sound on MCD. (Right) The influence of spatial sound on presence.

In addition to MCD difference, Figure 11 (Right) shows the presence score for Lab Replica VR and No Sound VR from the SUS questionnaire. There Lab Replica VR had slightly higher presence score than the No Sound VR, in line with previous research [9]. However, a logistic regression showed no significance in presence between Lab Replica and No Sound, $X^{2}(1, N=40)=.42, p=.52$.
To conclude, the results did not provide support for H5, suggesting that the addition of spatial sound in VR does not have a significant influence on MCD.

\section{DISCUSSION}

Results from the study indicated that people prefer a larger personal space with the robot in VR than in Live, confirming H1. It is also found that people perceived the virtual robot to be significantly more discomforting and have significantly lower feeling of presence in VR, confirming $\mathrm{H} 2$ and $\mathrm{H} 3$.

One explanation for the larger personal space with the robot in VR could be that distance perception is influenced by the HMD display. Previous research has shown that ego-centric distances are perceived as compressed in VEs [19][20], although no consensus has been drawn on the main cause of this effect [27]. Therefore, in this study, the personal space of the participants might have been bigger in VR than in Live to compensate for the distance compression in VR. Moreover, the study conducted by $\mathrm{x}$ [10] suggest that proxemics remain consistent in CAVE VR. Compared to HMD VR which was used in this research, CAVE systems have a larger field of view[28], a further distance between the display and the user [29], self-embodiment [30] which could have caused better distance perception. To further evaluate the potential of HMD VR in HRI, research should be done to investigate if distances compression in HMD VR can be circumvented by altering the virtual experience design. For example, Steinicke et al. [31] have shown that users significantly improve their distance estimation skills when they enter the virtual world via a transitional environment. Kelly et al. [32] suggest that allowing the participants to walk in the VE can help them to rescale the virtual space and improve their judgment accuracy.

Another reason why the participants kept the VR robot further away could be that the robot was perceived as more discomforting in VR. The increased feeling of discomfort might be caused by the quality of the 3D model, as it was not as realistic as the real robot. An alternative reason could be that, as the spatial sound was provided by the real movement of the robot and participants were wearing HMD, they could not see whether the real robot was moving or not. However, many mentioned that they felt that the real robot was moving and that might have caused them to feel more unsure and unsafe about the distance in VR. Future work with a more realistic $3 \mathrm{D}$ model of the robot or with computer generated spatial sound may reveal additional findings in the relationship between Live and VR. Multiple participants also mentioned that the VR robot felt more powerful, they explained that since they could not come into contact with robots in their daily life but had seen robots in a virtual setting (e.g. movie, games). Therefore, they considered robots belong to the virtual world and 
more powerful in VR. Future work can be done related to the qualitative evaluation of the responses of the participants, to not only understand their proxemic preferences, but also why they chose that. Additional work can be done to evaluate how previous experience with robots and exposure to VR can influence the relationship between Live and VR as well.

Results suggested that the addition of visual familiarity and spatial sound in VR has no significant influence on proxemic preferences nor the feeling of presence, rejecting $\mathrm{H} 4$ and $\mathrm{H} 5$. Our results related to presence were not in line with previous research, which suggested that the increase in visual familiarity can increase presence [18]. A possible explanation is that even though the VE was made to replicate the Live environment, there were still details that didn't correspond to each other. Participants could observe more visual inconsistency when the VE is similar with the Live environment than when the VE that is completely different from Live. Therefore, the addition of visual familiarity did not help to increase presence, and further research (with an even more realistic virtual environment) is needed to better understand this effect. Our results also showed that the addition of spatial sound did not increase the sense of presence in VR, another finding that deviates from previous research [9]. One reason could be that the researcher was giving oral instructions in VR, some participants mentioned that the instructions of the researcher pulled them out of the virtual experience. Further research should be done to investigate how to instruct experiments in VR without the researcher being socially present, e.g. through a virtual avatar or notification messages.

In general, this study suggests that HRI proxemics are shown to be different between Live and VR. However, results in different VR settings remain consistent with each other, indicating freedom within VR HRI itself to be used as a fast-prototyping tool. This finding should not stop the usage of VR in HRI. On the contrary, as VR HRI is more intended to be a more efficient prototyping tool or data collection tool, iterations can be done to first fine tune the HRI experience in VR before testing it in reality. On the other hand, the differences between Live and VR should be acknowledged, quantified or even circumvented through experiment design in future research.

\section{LIMITATION}

One limitation of this study was the spatial sound, which was created by moving the real robot. As participants were wearing HMD and were uncertain whether the real robot was moving or not, the trust in VR was influenced and thus may influence the experiment result. Another factor that limited this research was the social presence of the researcher in VR. As the participants were immersed in VR while receiving instructions from the researcher, they might have felt being pulled outside of VR.

\section{CONCLUSION}

This study compared Live and VR HRI with a focus on proxemics. VR settings with different visual familiarity and spatial sound were compared as well. Results indicated that proxemic preferences do not remain consistent between Live and HMD VR. However, the difference in proxemics could be caused by the compression of egocentric distance in VR. These differences in proxemics should be considered when conduction VR HRI experiments. Our comparison between Live and HMD VR suggested that the differences in the perception of the robot between Live and VR might have an influence on the task performance as well. Therefore, when conducting HRI research in VR, the different perceptions of the robot between Live and VR need to be compared before measuring the interaction. Moreover, it is also showed that results from different VR settings were consistent with each other, despite the absence of visual familiarity or spatial sound. This indicates more freedom to conduct VR HRI study as the result within VR itself tend to stay consistent, although more research needs to be done to confirm this finding.

\section{ACKNOWLEDGEMENTS}

The author would like to express sincere gratitude to Iolanda Leite for her supervision and guidance during the project. Additionally, the author would like to thank Sanne Van Waveren for her comments and insights and Marc van Almkerk for his valuable contribution to the technical implementation of the experiment.

\section{REFERENCES}

[1] T. Zhang et al., "Deep Imitation Learning for Complex Manipulation Tasks from Virtual Reality Teleoperation," ArXiv171004615 Cs, Oct. 2017.

[2] D. Whitney, E. Rosen, and S. Tellex, "Learning from Crowdsourced Virtual Reality Demonstrations," presented at the VAM-HRI 2018, Chicago, Illinois, USA, p. 3.

[3] T. Inamura and J. T. C. Tan, "Long-term large scale human-robot interaction platform through immersive VR system - Development of RoboCup @Home Simulator-," in 2012 IEEE/SICE International Symposium on System Integration (SII), 2012, pp. 242-247.

[4] D. Goedicke, J. Li, V. Evers, and W. Ju, "VROOM: Virtual Reality On-rOad Driving siMulation," in Proceedings of the 2018 CHI Conference on Human Factors in Computing Systems, New York, NY, USA, 2018, pp. 165:1-165:11.

[5] O. Liu, D. Rakita, B. Mutlu, and M. Gleicher, "Understanding human-robot interaction in virtual reality," 2017, pp. 751-757. 
[6] M. J. Schuemie, P. van der Straaten, M. Krijn, and C. A. P. G. van der Mast, "Research on Presence in Virtual Reality: A Survey," Cyberpsychol. Behav., vol. 4, no. 2, pp. 183201, Apr. 2001.

[7] The Hidden Dimension by Edward T. Hall. .

[8] V. Interrante, B. Ries, and L. Anderson, "Distance Perception in Immersive Virtual Environments, Revisited," in IEEE Virtual Reality Conference (VR 2006), 2006, pp. 3-10.

[9] M. Kobayashi, K. Ueno, and S. Ise, "The Effects of Spatialized Sounds on the Sense of Presence in Auditory Virtual Environments: A Psychological and Physiological Study," Presence, vol. 24, no. 2, pp. 163-174, May 2015.

[10] H. Kamide, Y. Mae, T. Takubo, K. Ohara, and T. Arai, "Direct comparison of psychological evaluation between virtual and real humanoids: Personal space and subjective impressions," Int. J. Hum.-Comput. Stud., vol. 72, no. 5, pp. 451-459, May 2014.

[11] M. Bennett, T. Williams, D. Thames, and M. Scheutz, "Differences in interaction patterns and perception for teleoperated and autonomous humanoid robots," in 2017 IEEE/RSJ International Conference on Intelligent Robots and Systems (IROS), 2017, pp. 6589-6594.

[12] A. Sardar, M. Joosse, A. Weiss, and V. Evers, "Don'T Stand So Close to Me: Users' Attitudinal and Behavioral Responses to Personal Space Invasion by Robots," in Proceedings of the Seventh Annual ACM/IEEE International Conference on Human-Robot Interaction, New York, NY, USA, 2012, pp. 229-230.

[13] L. Takayama and C. Pantofaru, "Influences on proxemic behaviors in human-robot interaction," in 2009 IEEE/RSJ International Conference on Intelligent Robots and Systems, 2009, pp. 5495-5502.

[14] J. Mumm and B. Mutlu, "Human-robot Proxemics: Physical and Psychological Distancing in Human-robot Interaction," in Proceedings of the 6th International Conference on Human-robot Interaction, New York, NY, USA, 2011, pp. 331-338.

[15] C. Peters, F. Yang, H. Saikia, C. Li, and G. Skantze, "Towards the use of Mixed Reality for HRI Design via Virtual Robots," presented at the VAM-HRI'18, Chicago USA, 2018.

[16] H. Huettenrauch, K. S. Eklundh, A. Green, and E. A. Topp, "Investigating Spatial Relationships in Human-Robot Interaction," in 2006 IEEE/RSJ International Conference on Intelligent Robots and Systems, 2006, pp. 5052-5059.

[17] Joy van Baren and Wijnand IJsselsteijn, "Measuring Presence: A Guide to Current
Measurement Approaches," OmniPres project IST-2001-39237, Mar. 2004.

[18] M. Usoh, E. Catena, S. Arman, and M. Slater, "Using Presence Questionnaires in Reality," Presence Teleoperators Virtual Environ., vol. 9, no. 5, pp. 497-503, Oct. 2000.

[19] A. A. Gooch and P. Willemsen, "Evaluating Space Perception in NPR Immersive Environments," in Proceedings of the $2 \mathrm{Nd}$ International Symposium on Nonphotorealistic Animation and Rendering, New York, NY, USA, 2002, pp. 105-110.

[20] R. Messing and F. H. Durgin, "Distance Perception and the Visual Horizon in HeadMounted Displays," ACM Trans Appl Percept, vol. 2, no. 3, pp. 234-250, Jul. 2005.

[21] C. Hendrix and W. Barfield, "The Sense of Presence within Auditory Virtual Environments," Presence Teleoperators Virtual Environ., vol. 5, no. 3, pp. 290-301, Jan. 1996.

[22] H. Q. Dinh, N. Walker, L. F. Hodges, C. Song, and A. Kobayashi, "Evaluating the importance of multi-sensory input on memory and the sense of presence in virtual environments," in Proceedings IEEE Virtual Reality (Cat. No. 99CB36316), 1999, pp. 222-228.

[23] D. R. Begault, 3-D Sound for Virtual Reality and Multimedia. San Diego, CA, USA: Academic Press Professional, Inc., 1994.

[24] A. F. Kinzel, "Body-buffer zone in violent prisoners," Am. J. Psychiatry, vol. 127, no. 1, pp. 59-64, Jul. 1970.

[25] C. M. Carpinella, A. B. Wyman, M. A. Perez, and S. J. Stroessner, "The Robotic Social Attributes Scale (RoSAS): Development and Validation," in Proceedings of the 2017 ACM/IEEE International Conference on Human-Robot Interaction, New York, NY, USA, 2017, pp. 254-262.

[26] M. Slater, M. Usoh, and A. Steed, "Taking Steps: The Influence of a Walking Technique on Presence in Virtual Reality," ACM Trans Comput-Hum Interact, vol. 2, no. 3, pp. 201219, Sep. 1995.

[27] C. J. Lin and B. H. Woldegiorgis, "Interaction and visual performance in stereoscopic displays: A review," J. Soc. Inf. Disp., vol. 23, no. 7, pp. 319-332.

[28] P. Willemsen, M. B. Colton, S. H. CreemRegehr, and W. B. Thompson, "The Effects of Head-mounted Display Mechanical Properties and Field of View on Distance Judgments in Virtual Environments," ACM Trans Appl Percept, vol. 6, no. 2, pp. 8:1-8:14, Mar. 2009.

[29] G. Bruder, F. Argelaguet, A.-H. Olivier, and A. Lécuyer, "CAVE Size Matters: Effects of Screen Distance and Parallax on Distance Estimation in Large Immersive Display 
Setups," Presence Teleoperators Virtual Environ., vol. 25, no. 1, pp. 1-16, May 2016.

[30] B. J. Mohler, S. H. Creem-Regehr, W. B. Thompson, and H. H. Bülthoff, "The Effect of Viewing a Self-Avatar on Distance Judgments in an HMD-Based Virtual Environment," Presence Teleoperators Virtual Environ., vol. 19, no. 3, pp. 230-242, Jun. 2010.

[31] F. Steinicke, G. Bruder, K. Hinrichs, M. Lappe, B. Ries, and V. Interrante, "Transitional Environments Enhance Distance Perception in Immersive Virtual Reality Systems," in Proceedings of the 6th Symposium on Applied Perception in Graphics and Visualization, New York, NY, USA, 2009, pp. 19-26.

[32] J. W. Kelly, L. S. Donaldson, L. A. Sjolund, and J. B. Freiberg, "More than just perceptionaction recalibration: Walking through a virtual environment causes rescaling of perceived space," Atten. Percept. Psychophys., vol. 75, no. 7, pp. 1473-1485, Oct. 2013.

\section{APPENDIX 1}

\section{SUS questionnaire: VR}

Please rate your sense of being in the office space, on the following scale from 1 to 7 , where 7 represents your normal experience of being in a place.

I had a sense of "being there" in the Virtual Environment:

1. Not at all ... 7. Very much.

2. To what extent were there times during the experience when the Virtual Environment was the reality for you?

There were times during the experience when the Virtual Environment was the reality for me..

$$
\text { 1. At no time ... 7. Almost all the time. }
$$

3. When you think back about your experience, do you think of the Virtual Environment more as images that you saw, or more as somewhere that you visited? The Virtual Environment seems to me to be more like...

1. Images that I saw ... 7. Somewhere that I visited.

4. During the time of the experience, which was strongest on the whole, your sense of being in the Virtual Environment, or of being elsewhere?

I had a stronger sense of...

1. Being elsewhere ... 7. Being in the Virtual Environment.

5. Consider your memory of being in the Virtual Environment. How similar in terms of the structure of the memory is this to the structure of the memory of other places you have been today? By 'structure of the memory' consider things like the extent to which you have a visual memory of the Virtual Environment, whether that memory is in colour, the extent to which the memory seems vivid or realistic, its size, location in your imagination, the extent to which it is panoramic in your imagination, and other such structural elements. I think of the Virtual Environment as a place in a way similar to other places that I've been today...

$$
\text { 1. Not at all ... 7. Very much so. }
$$

6. During the time of the experience, did you often think to yourself that you were actually in the Virtual Environment? During the experience, I often thought that I was really standing in the Virtual Environment...

1. Not very often ... 7. Very much so. 

www.kth.se 